\title{
Focused Screening of a Panel of Cancer-Related Genetic Polymorphisms Reveals New Susceptibility Loci for Pediatric Acute Lymphoblastic Leukemia
}

\author{
Sonja Offenmüller, ${ }^{1}$ Yadddanapudi Ravindranath, $\mathrm{MBBS}^{2}{ }^{2}$ Gerard Goyette, $\mathrm{BS}^{2}{ }^{2}$ Deepa Kanakapalli, $\mathrm{MA}^{2}$ \\ Kathryn S. Miller, BSN, ${ }^{2}$ Ines B. Brecht, $\mathrm{MD}^{1,2 *}$ and Oliver Zolk, MD, $\mathrm{PhD}^{3,4}$
}

\begin{abstract}
Background. A genetic predisposition to acute lymphoblastic leukemia (ALL) in childhood is well established. Currently known risk loci, however, explain only one third of the estimated total risk related to common genetic variations. Procedure. We genotyped 1,421 polymorphisms in 407 candidate genes from the SNP500Cancer database (National Cancer Institute) using the Illumina Cancer SNP Panel. We investigated 78 cases (aged 0-19 years at diagnosis, and mixed ethnic background) of childhood Bprecursor ALL and compared genotype data with those of 1,417 HapMap controls. To account for the ethnic diversity of the study population, structured association by genetically matching cases and controls using identity-by-state similarity was used. Case-control association analyses were performed using Cochran-Mantel-
\end{abstract}

Haenszel tests, adjusted for the population substructure. Results. Common variations rs6966 ( $3^{\prime}$ UTR of PPP1R13L, chr 19q13.32, $P=4.55 \times 10^{-9}$ ) and rs414580 (intron 2 of MSR1, chr 8p22, $P=6.09 \times 10^{-8}$ ) were significantly associated with ALL. These SNPs remained significant after adjustment for multiple testing. The SNP rs6966 tags a haplotype block which includes SNPs in PPP1R13L and ERCC2 genes, which are related to DNA repair and cell survival. rs6966 and rs414580 conferred allelic odds ratios of $3.74(95 \%$ confidence interval $[\mathrm{Cl}] 2.31-6.04)$ and $3.93(95 \% \mathrm{Cl}$ 2.31-6.69), respectively. Conclusions. These findings reveal two independent novel susceptibility loci for childhood ALL. Pediatr Blood Cancer 2014;61:1411-1415. (C) 2014 Wiley Periodicals, Inc.

Key words: candidate gene association study; childhood acute lymphoblastic leukemia; ERCC2; MSR1; PPP1R13L

\section{INTRODUCTION}

Acute lymphoblastic leukemia (ALL) represents almost one third of pediatric cancer diagnoses, making it the most common malignancy in childhood [1]. ALL is most commonly of the B cell lineage and its incidence peaks between ages 2 and 4 years. B-precursor ALL (B-ALL) — despite the high rate of cure-remains one of the leading causes of cancer-related death among children [2]. Somatically acquired genetic aberrations in ALL lymphoblasts are prognostic and can guide risk-directed therapy. The extent to which germline variation contributes to ALL susceptibility, however, is less clear and is subject of current research.

Direct evidence for a genetic predisposition to ALL is provided by the high risk associated with Bloom syndrome, neurofibromatosis, ataxia teleangiectasia, and Down's syndrome. The heritable basis of susceptibility to ALL is further supported by recent candidate gene (CGAS) and genome-wide association studies (GWAS), suggesting that co-inheritance of multiple germline variants may contribute to disease risk. Five GWAS have been performed so far with populations between 50/50 and 3,275/4,817 ALL cases/healthy control individuals [3-7]. These studies identified several risk loci with allelic odds ratios (OR) of the disease-related allele between 1.34 and 9.99.

Enciso-Mora et al. [8] calculated that $25 \%$ of the total variation in B-ALL risk is accounted for by common genetic variation. On the other hand, previous GWAS-identified loci (IKZF1, CDKN2A, $A R I D 5 B$, and $C E B P E$ ) explain only $8 \%$ of this total. The data provide the rationale for continued investigation of additional susceptibility loci that were likely missed by previous GWAS. Although GWAS represent a powerful approach to the identification of disease loci, the $P$-value requirement for defining a significant association, in turn, may increase the probability of missing a true association [9].

In the present study we, therefore, have chosen a CGAS approach which was based on the assumption that known risk loci for many types of cancer are a priori also potential risk loci for B-ALL. Single nucleotide polymorphisms (SNPs) were selected from the SNP500Cancer database, which is biased towards SNPs that lie within or are situated close to cancer "candidate" genes [10]. We genotyped 1,421 polymorphisms in 407 candidate genes using the Illumina Cancer SNP Panel BeadChip assay (Illumina, San Diego, CA). We investigated an unselected population of B-ALL patients recruited in a single center irrespective of ethnic background to identify risk loci independent of ethnicity.

\section{METHODS}

\section{Clinical Samples}

Patients diagnosed with acute lymphoblastic leukemia (ALL) who were diagnosed and treated at the Children's Hospital of

Additional Supporting Information may be found in the online version of this article at the publisher's web-site.

${ }^{1}$ Pediatric Oncology and Hematology, University Children's Hospital, Erlangen, Germany; ${ }^{2}$ Georgie Ginopolis Chair for Pediatric Cancer and Blood Diseases, Children's Hospital of Michigan and Wayne State University School of Medicine, Detroit, Michigan; ${ }^{3}$ Institute of Experimental and Clinical Pharmacology and Toxicology, FriedrichAlexander-Universität Erlangen-Nürnberg, Erlangen, Germany; ${ }^{4}$ Institute of Pharmacology of Natural Products and Clinical Pharmacology, University of Ulm, Ulm, Germany

Grant sponsor: Kind-Philipp-Stiftung für Leukämieforschung (S.O.); Grant sponsor: Leukemia Research; Grant sponsor: Life Inc.; Grant sponsor: Melissa Krinsky Ginoplois-Karmanos Endowment (Y.R.); Grant sponsor: Georgie Ginopolis Chair endowment Award; Grant sponsor: Leukemia Research Life, Inc.

Conflict of interest: Nothing to declare.

Brecht and Zolk share senior authorship.

*Correspondence to: Ines B. Brecht, Pediatric Oncology and Hematology, University Children's Hospital, Loschgestrasse 15, Erlangen 91054, Germany. E-mail: ines.brecht@uk-erlangen.de

Received 29 August 2013; Accepted 3 February 2014 
Michigan, Detroit, USA between 1993 and 2007 were screened for inclusion. Genomic DNA was extracted from discarded blood samples obtained for routine clinical hematology studies. All samples were anonymized prior to testing. The research protocol was approved by the Human Investigation Committee of Wayne State University School of Medicine.

Seventy-eight children with B-precursor ALL who had finished the treatment protocols and had remission blood or bone marrow samples available were recruited. Patient characteristics are summarized in Table I. Genomic DNA was extracted from the samples using Qiagen genomic DNA extraction kits (QIAGEN, Inc., Valencia, CA). Quantity and quality of the gDNA was evaluated by using Molecular Probes PicoGreen Assay (Molecular Probes, Eugene, OR).

\section{SNP Selection, Genotyping, and Quality Control}

Genotyping was performed using the Illumina Cancer SNP Panel on the GoldenGate Assay system according to the manufacturer's instructions (Illumina). The Cancer Panel contains 1,421 SNPs in 407 hypothesized cancer-related genes from the SNP500Cancer database (Cancer Genome Anatomy Project, National Cancer Institute). SNPs were chosen from public databases and reports, and the choice of genes includeds a bias towards non-synonymous and promoter SNPs in genes that have been implicated in one or more cancers (PMID 16381944, 14681474). The Illumina Cancer SNP Panel includes genes of several cancer-related pathways, such as oncogenesis, tumor suppression, oxidative and hypoxic stress, detoxification, DNA mismatch repair, regulation of transcription and cell cycle, metabolism and immune regulation, and different carriers and transport proteins. Genes and pathways covered by the Cancer SNP Panel are shown in the Supplemental Table I. In the Cancer SNP Panel, more than three SNP assays were selected, on average, for each gene represented. It was not the primary intention of this study

TABLE I. Patient and Biological Characteristics

\begin{tabular}{lc}
\hline & Cases $(\mathrm{n}=78)$ \\
\hline Sex & \\
$\quad$ Male & $44(56.4 \%)$ \\
Female & $34(43.6 \%)$ \\
Race & \\
$\quad$ African Americans & $13(16.7 \%)$ \\
Asian Americans \& Pacific Islander Americans & $4(5.1 \%)$ \\
Indian Americans & $3(3.8 \%)$ \\
Caucasians & $58(74.4 \%)$ \\
Age at diagnosis & \\
$\quad<10$ years & $62(79.5 \%)$ \\
$\quad \geq 10$ years & $16(20.5 \%)$ \\
Immunophenotype & \\
B-precursor ALL & $74(94.8 \%)$ \\
Mixed lineage ALL & $2(2.6 \%)$ \\
No information & $2(2.6 \%)$ \\
TEL/AML1 & $16(20.5 \%)$ \\
Positive & $62(79.5 \%)$ \\
Negative or no information & \\
MLL/AF4 & $1(1.3 \%)$ \\
Positive & $77(98.7 \%)$ \\
Negative or no information & \\
\hline
\end{tabular}

Pediatr Blood Cancer DOI 10.1002/pbc to replicate previous GWAS or CGAS results but to find novel candidates. In fact, there is only a small overlap between SNPs and genes covered by the Illumina Cancer SNP Panel and loci identified in previous association studies. For example, $56 \%$ of the genes and $22 \%$ of the SNPs identified in the meta-analysis of CGAS by Vijayakrishnan et al. [11] were included in the Cancer SNP Panel.

Mathematical clustering and automatic genotype calling algorithms, implemented in the GenomeStudio software (Illumina), was used to analyze the raw intensity data. To assess the genotype quality, we manually inspected the cluster plots and corrected genotype assignment where automatic genotype calling failed.

SNPs that could not be clustered or were non-polymorphic were excluded from further analyses. The average genotyping call rate for all samples was $98.8 \%$. If the call rate for any sample was below $95 \%$, the sample was excluded from further analysis. Other quality criteria included minor allele frequency (MAF) $\geq 0.01$ and $P$-value for the Hardy-Weinberg equilibrium test $>0.01$. Forty-two SNPs were removed during quality control, leaving 1,387 SNPs for analysis.

To exclude genotyping artifacts, candidate SNPs were genotyped with an independent technology. We used the TaqMan platform and predesigned SNP Genotyping Assays Reagents (Applied Biosystems, Foster City, CA) to re-genotype rs6966 (C_2615637_10) and rs414580 (C_1865305_10) as described before [12].

We used the HapMap population $(\mathrm{N}=1,417)$ as a control. Genotype data were retrieved from the publicly accessible HapMap database (1,417 samples; HapMap Genome Browser release \#28, The International Hapmap Project, available at http://hapmap.ncbi. nlm.nih.gov/; genotype data were available for 1,169 SNPs included in the Illumina Cancer SNP Panel) and from Illumina (subset of 269 CEU, CHB/JPT, and YRI samples from the HapMap population, genotype data were available for all 1,421 SNPs).

\section{Statistical Analysis}

To adjust for inflation due to population stratification, structured association by genetically matching cases and controls using identity-by-state (IBS) similarity as implemented in PLINK [13] was used. Complete linkage agglomerative clustering was performed, based on pairwise IBS distance, with some modifications to the clustering process: restrictions based on a significance test for whether two individuals belong to the same population (i.e., do not merge clusters that contain significantly different individua1s), a phenotype criterion (i.e., all clusters must contain at least one ALL case and one control) and cluster size restrictions (i.e., such that, with a cluster size of two, the subsequent association test would implicitly match every case with its nearest control, as long as the case and control do not show evidence of belonging to different populations). Based on the matrix of IBS pairwise distances, multi-dimensional scaling (MDS) components were obtained using the MDS-plot option in PLINK. MDS plots, generated with $\mathrm{R}$, visualize substructure and provide quantitative indices of population genetic variation. Stratified case-control association analyses were performed using Cochran-MantelHaenszel tests, adjusted for the population substructure by inclusion of the IBS cluster information. All the statistical results are reported without correction, unless indicated otherwise. Control of the error rate under multiple testing was done using false discovery rate (FDR) adjustment as implemented in PLINK. After exclusion of 
some SNPs after quality control and based on the LD structure of cases and controls, we calculated the effective number of independent tests in our analysis at 1,379. Power analysis was done with PS Power and Sample Size v3.0 [14].

\section{RESULTS}

Before comparing allele frequencies between cases and controls, we considered biases due to nonrandom distribution of technical artifacts or to population differences between case and control data, which would result in a non-null distribution of test statistics with excess false-positive associations. Therefore, we assessed the median distribution of test statistics with the genomiccontrol parameter $\lambda_{\mathrm{GC}}$ and examined the tail of the distribution of association statistics in a comparison of observed and expected $P$-values (Q-Q plot; Fig. 1). After adjustment for inflation due to population stratification, $\lambda_{\mathrm{GC}}$ was markedly reduced, falling from 5.86 to 1.29 . The MDS plot shows clustering of cases within the major control populations (Fig. 2).

To exclude genotyping artifacts, candidate SNPs were genotyped with an independent technology. The concordance of genotype calls was greater than $98 \%$, indicating that the extreme tail of low $P$-values was not substantially contaminated by genotyping artifacts.

Our analysis revealed a significant association of the (noncoding) haplotype-tagging SNPs rs6966 (NM_001142502.1: c. *486A $>\mathrm{T}$, chr $19 \mathrm{q} 13.3)$ in the protein phosphatase 1 regulatory subunit

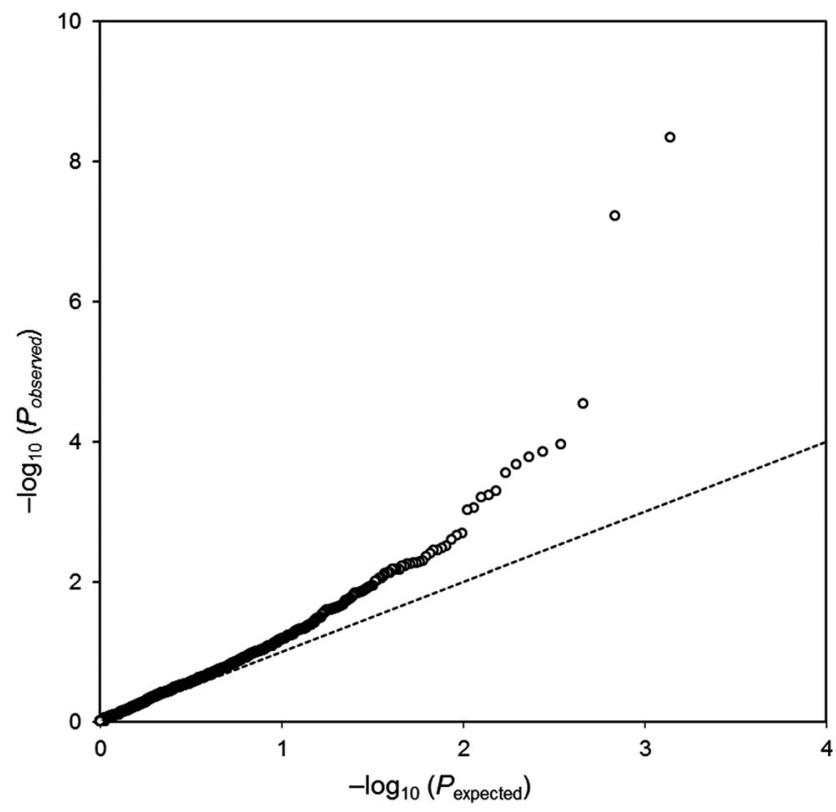

Fig. 1. Q-Q plot for the association of 1,387 tested SNPs and the ALL risk after controlling the study population (1,417 controls and 78 ALL cases) for confounding by ethnicity (identity-by-state clustering approach). The Q-Q plot allows assessment of the number and magnitude of observed associations between genotyped SNPs and ALL, compared to the association statistics expected under the null hypothesis of no association.

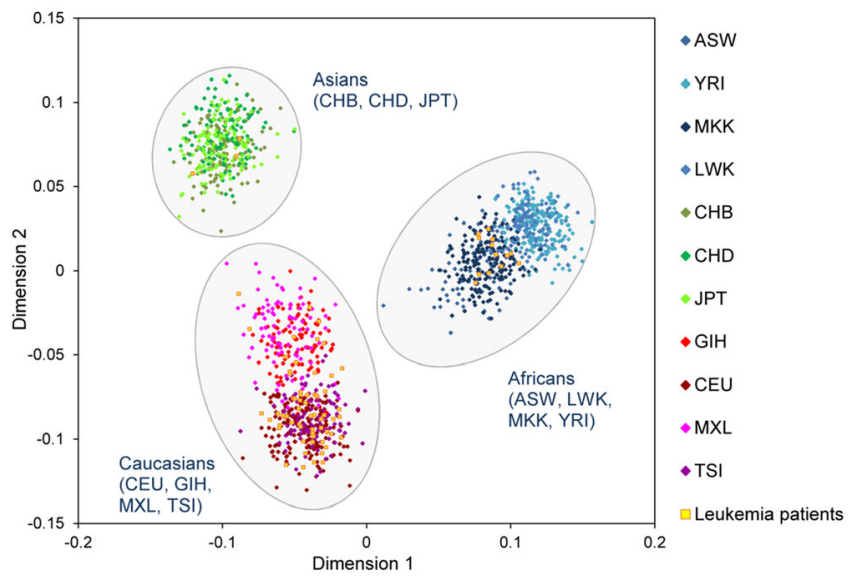

Fig. 2. Multidimensional scaling plot of the identity by state matrix.

13 like $(P P P 1 R 13 L)$ gene $\left(P=4.55 \times 10^{-9}\right)$ and $\mathrm{rs} 414580$ (NM_002445.3: c. $103+93 \mathrm{~A}>\mathrm{T}$, chr 8p22) in the macrophage scavenger receptor $1(M S R 1)$ gene $\left(P=6.09 \times 10^{-8}\right)$ with ALL (Fig. 3). For both SNPs, the respective T alleles were associated with increased ALL risk. A third SNP, rs 11762213 (NM_000245.2: c. $144 \mathrm{G}>\mathrm{A}, \mathrm{chr} 7 \mathrm{q} 31)$ in the MET proto-oncogene (MET), showed only borderline significance $\left(P=2.97 \times 10^{-2}\right.$ after correction for multiple testing). The SNPs rs6966 in PPP1R13L, rs414580 in $M S R 1$, and rs11762213 in MET conferred allelic odds ratios of 3.74 (95\% CI 2.31-6.04), 3.93 (95\% CI 2.31-6.69), and 7.57 (95\% CI 2.52-22.78), respectively. MAFs in cases/controls of the total population were 0.77/0.46 (rs414580), 0.77/0.47 (rs6966), and 0.23/0.03 (rs11762213). MAFs in cases/controls in the Caucasian subpopulations $(\mathrm{N}=64 / 463)$ were $0.77 / 0.40$ ( $\mathrm{rs} 414580), 0.85 / 0.35$ (rs6966), and 0.23/0.04 (rs11762213).

Based on HapMap data, haplotype block organization in the rs6966 and rs414580 regions was investigated using criteria based on solid spine of linkage disequilibrium method $\left(\mathrm{D}^{\prime}>0.8\right)$ with Haploview software. Figure 4 shows LD maps in the HapMap European (CEU/TSI) population (LD plots for Asian and African populations are shown in Supplemental Figs. 1 and 2). The SNP rs6966 was in LD with SNPs in PPP1R13L as well as excision repair cross-complementing rodent repair deficiencycomplementation group 2 (ERCC2).

In addition to identifying new loci associated with ALL risk, we sought to replicate previous positive associations. Six SNPs previously linked in genome-wide or candidate gene association studies with ALL risk were included in the Illumina SNP Cancer Panel, namely $C D K N 2 A$ rs 3731217 ( $P=0.1022$; OR 0.54), EPHX1 rs1051740 ( $P=0.3648$; OR 0.77), $M T H F R$ rs $1801133(P=0.7829$; OR 0.93), MTR rs1805087 $(P=0.1390 ;$ OR 0.62), SLC19A1 rs1051266 $(P=0.7984 ;$ OR 0.93), and XRCCl rs25487 $(P=0.3248$; OR 1.30) [11,15-18]. The respective genotype data were thus available for analysis. None of these SNPs were significantly (after correction for multiple testing) associated with ALL in our study. It is important to note that, because our study was powered to detect an OR $>2.3$ (power of $>80 \%$ to detect associations with $\mathrm{OR}>2.3$ at a nominal significance level of $3.5 \times 10^{-5}$ and a minor allele frequency of 0.3 ), we cannot exclude that these SNPs are significantly associated with ALL at a lower OR level. 


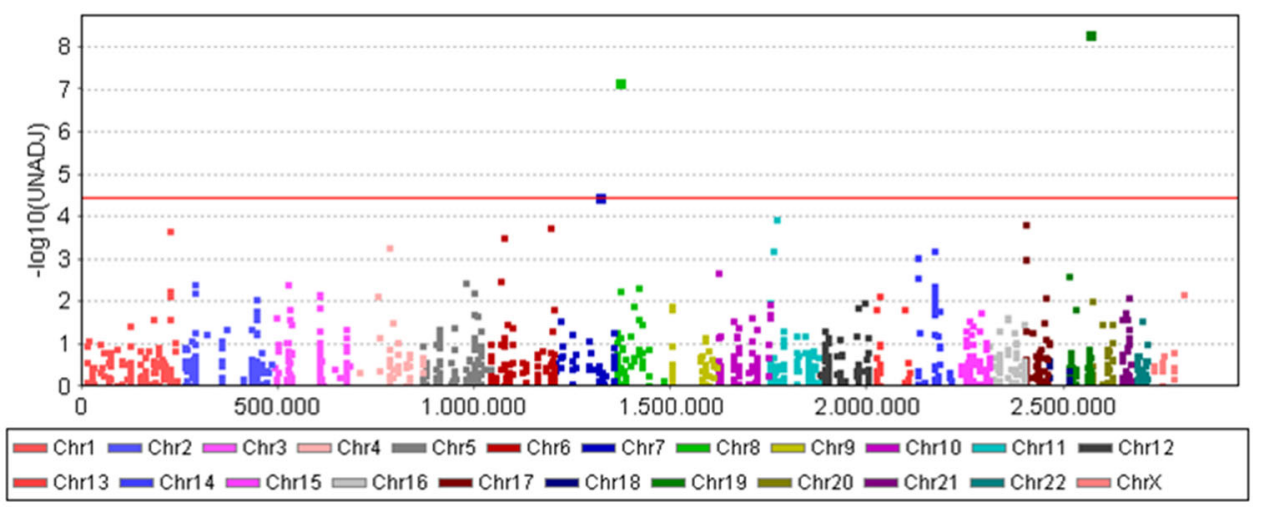

Fig. 3. Manhattan plot showing the association of SNPs with ALL. The $P$-values $\left(-\log _{10} P\right)$ plotted against their respective positions on each chromosome. Each chromosome is depicted in a different color. $-\log _{10}$ (UNADJ), $-\log _{10} P$ value of Cochran-Mantel-Haenszel test unadjusted for multiple testing.

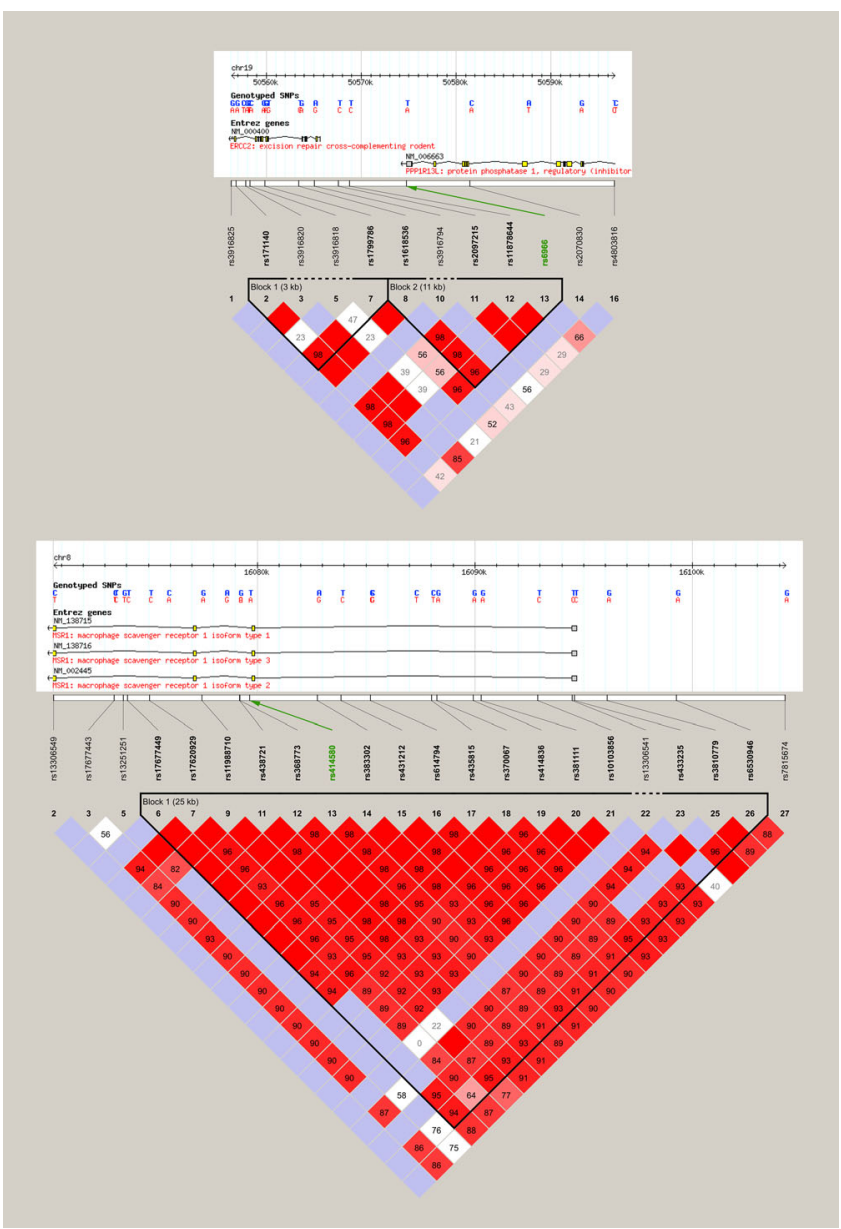

Fig. 4. LD maps of the regions adjacent to rs6966 in $P P P 1 R 13 L$ and rs414580 in MSR1 in European (CEU/TSI) populations. The SNPs rs6966 and rs414580 are highlighted in green. Shading reflects differences in pairwise LD (white $\mathrm{r}^{2}=$ low LD; red $\mathrm{r}^{2}=$ near-perfect LD). Numbers in squires are estimates of pairwise coefficients $\left(\mathrm{D}^{\prime}\right)$, expressed in percentages. Unreported values reflect $\mathrm{D}^{\prime}$ of $1.0(100 \%)$.

\section{DISCUSSION}

This is the first study evaluating an association of SNPs in "cancer genes" from the SNP500 database with childhood ALL. Similar analyses using the Illumina Cancer SNP Panel were successfully applied previously to identify risk loci for example for colorectal or prostate cancer $[19,20]$.

The SNPs identified in our analysis, rs6966, rs414580, and rs11762213, have not been associated previously with childhood ALL in GWAS or CGAS. The tag SNP rs6966, located on chromosome $19 \mathrm{q} 13.3$, is in LD with polymorphisms in ERCC2 and PPP1R13L, genes related to DNA repair and cell survival. PPP1R13L induces apoptosis by blocking NFKB or inhibits apoptosis by blocking P53 [21,22]. By either mechanism, the gene could influence the survival of precancerous lesions, and it has been speculated that $P P P 1 R 13 L$ participates in the tumorigenesis process [22-24]. A first link between PPP1R13L and ALL was provided by the observation that $P P P 1 R 13 L$ gene expression was significantly higher in cells of acute leukemia than that in cells from healthy donors or acute leukemia patients in complete remission [25].

The $5^{\prime} \rightarrow 3^{\prime}$ helicase protein ERCC2 (also known as XPD) has an important role in the nucleotide excision repair (NER) pathway [26]. . The NER pathway plays a major role in cell protection against genotoxic damage by repairing DNA lesions such as those induced by UV irradiation or chemical carcinogens [27]. Inherited variations in DNA-repair efficiency have been implicated in the predisposition to de novo and therapy-related AML [28,29]. Moreover, Hernandez-Boluda et al. [29] identified rs13181 in ERCC2 as an independent risk factor for leukemic transformation in primary myelofibrosis, even though their finding is not without controversy [30,31]. Several reports linked polymorphisms in the ERCC2 gene with enhanced risk of childhood ALL [32,33], although negative results were also reported [34,35]. A meta-analysis was able to confirm an association of the ERCC2 locus with ALL [26].

We identified rs6966 as a potential susceptibility locus, but it remains a challenge to pinpoint the causal variant, which may be located in PPP1R13L or ERCC2. Both genes have been linked with tumorigenesis or leukemic transformation and thus may play a role in the development of ALL. 
The other tag SNP identified in our study, rs414580, is located in the 8 p22 region in the $M S R 1$ gene [36]. Genetic polymorphisms in MSR1 have been shown to be related to prostate cancer risk, although the underlying mechanism is unclear [37]. In a mouse chronic myeloid leukemia (CML) model, Chen et al. [38] demonstrated that MSR1 suppresses leukemia stem cells and CML development. Interestingly, the ALL susceptibility loci MSR 1 (this study) and $C E B P E$ (previous studies [3,39],) both play a role in normal macrophage function [40,41].

The third significantly associated SNP in our analysis was rs11762213 in MET, although only at borderline significance. Germline mutations located in the MET proto-oncogene, which lead to a constitutive activation of the MET protein, have been linked with the development of hereditary papillary renal carcinoma [42]. Moreover, the germline genetic polymorphism rs11762213 has been associated with the risk of recurrence in patients with localized renal-cell carcinoma [43]. This SNP, however, has not been associated previously with childhood ALL.

Six SNPs previously linked with susceptibility to childhood ALL were included in our SNP panel. Previous studies showed that the effect size of these SNPs was small and associations became obvious, in some cases, only after combining results from different studies in meta-analyses (OR 1.1-1.4) [11,15-18]. Our study was powered to detect an OR $>2.3$, which explains the lack of association of these SNPs in our study.

Limitations of our study include the small sample size. Our study had statistical power of $>80 \%$ to detect only associations with OR $>2.3$ at a nominal significance level of $3.5 \times 10^{-5}$ (this significance threshold is required when accounting for multiple comparisons) and a minor allele frequency of 0.3. Nevertheless, this is the first study that systematically screened tagging and several coding variants in established cancer risk genes in a comprehensive manner to identify ALL susceptibility loci. The study was restricted to precursor B-ALL patients. Although the study design precludes any statements on the relevance of the identified risk loci for patients with the T-cell immunophenotype, restriction to B-ALL reduced the heterogeneity of the study population and thus improved the power. Patients were not selected according to their ethnic background, which on the one hand necessitated statistical adjustments but on the other hand enabled identification of susceptibility loci not restricted to only one major ethnic group.

In conclusion, by screening 1,421 SNPs in "cancer genes" we identified two novel loci, namely ERCC2/PPPIR13L and MSR1, which are potentially associated with susceptibility to childhood ALL. Confirmation of the association is required in an independent study population with greater statistical power. Rapid advances in the development of sequencing technologies will enable costeffective screening of several thousand clinically relevant SNPs in childhood ALL in the near future. As a proof-of-concept, Wesolowska et al. [9] applied a novel multiplexing method with custom-designed baits to screen 25,000 SNPs in childhood ALL.

\section{ACKNOWLEDGMENTS}

The present work was performed as part of the doctoral thesis of S.O. This work is supported by the German Kind-PhilippStiftung and the Georgie Ginopolis Chair endowment award to Dr. Ravindranath and a grant from Leukemia Research Life, Inc.; the authors also wish to acknowledge initial encouragement for the project by Dr. Richard Everson.

\section{REFERENCES}

1. Pui CH, Carroll WL, Meshinchi S, et al. Biology, risk stratification, and therapy of pediatric acute leukemias: An update. J Clin Oncol 2011:29:551-565.

2. Mullighan CG. Molecular genetics of B-precursor acute lymphoblastic leukemia. J Clin Invest 2012;122:3407-3415

3. Papaemmanuil E, Hosking FJ, Vijayakrishnan J, et al. Loci on 7p12.2, 10q21.2 and 14q11.2 are associated with risk of childhood acute lymphoblastic leukemia. Nat Genet 2009;41:1006-1010.

4. Trevino LR, Yang W, French D, et al. Germline genomic variants associated with childhood acute lymphoblastic leukemia. Nat Genet 2009;41:1001-1005.

5. Han S, Lee KM, Park SK, et al. Genome-wide association study of childhood acute lymphoblastic leukemia in Korea. Leukemia Res 2010;34:1271-1274.

6. Sherborne AL, Hosking FJ, Prasad RB, et al. Variation in CDKN2A at 9p21.3 influences childhood acute lymphoblastic leukemia risk. Nat Genet 2010;42:492-494.

7. Ellinghaus E, Stanulla M, Richter G, et al. Identification of germline susceptibility loci in ETV6-RUNX1rearranged childhood acute lymphoblastic leukemia. Leukemia 2012;26:902-909.

8. Enciso-Mora V, Hosking FJ, Sheridan E, et al. Common genetic variation contributes significantly to the risk of childhood B-cell precursor acute lymphoblastic leukemia. Leukemia 2012;26:2212-2215.

9. Wesolowska A, Dalgaard MD, Borst L, et al. Cost-effective multiplexing before capture allows screening of 25,000 clinically relevant SNPs in childhood acute lymphoblastic leukemia. Leukemia 2011;25:1001-1006.

10. Packer BR, Yeager M, Burdett L, et al. SNP500Cancer: A public resource for sequence validation, assay development, and frequency analysis for genetic variation in candidate genes. Nucl Acid Res 2006;34: D617-D621.

11. Vijayakrishnan J, Houlston RS. Candidate gene association studies and risk of childhood acute lymphoblastic leukemia: a systematic review and meta-analysis. Haematologica 2010;95:1405-1414.

12. Trenk D, Hochholzer W, Fromm MF, et al. Paraoxonase-1 Q192R polymorphism and antiplatelet effects of clopidogrel in patients undergoing elective coronary stent placement. Circ Cardiovasc Genet 2011; 4:429-436.

13. Purcell S, Neale B, Todd-Brown K, et al. PLINK: A tool set for whole-genome association and population-based linkage analyses. Am J Hum Genet 2007;81:559-575.

14. Dupont WD, Plummer WD, Jr. Power and sample size calculations. A review and computer program. Controlled Clin Trials 1990;11:116-128.

15. Wang $\mathrm{L}$, Yin $\mathrm{F}, \mathrm{Xu} \mathrm{X}$, et al. X-ray repair cross-complementing group 1 (XRCC1) genetic polymorphisms and risk of childhood acute lymphoblastic leukemia: A meta-analysis. PLoS ONE 2012;7:e34897.

16. Urayama KY, Chokkalingam AP, Manabe A, et al. Current evidence for an inherited genetic basis of childhood acute lymphoblastic leukemia. Int J Hematol 2013;97:3-19.

17. Wang H, Wang J, Zhao L, et al. Methylenetetrahydrofolate reductase polymorphisms and risk of acute lymphoblastic leukemia-evidence from an updated meta-analysis including 35 studies. BMC Med Genet 2012:13:77.

18. de Jonge R, Tissing WJ, Hooijberg JH, et al. Polymorphisms in folate-related genes and risk of pediatric acute lymphoblastic leukemia. Blood 2009;113:2284-2289.

19. Chen J, Pande M, Huang YJ, et al. Cell cycle-related genes as modifiers of age of onset of colorectal cancer in Lynch syndrome: A large-scale study in non-Hispanic white patients. Carcinogenesis 2013; 34:299-306.

20. Barwick BG, Abramovitz M, Kodani M, et al. Prostate cancer genes associated with TMPRSS2-ERG gene fusion and prognostic of biochemical recurrence in multiple cohorts. Br J Cancer 2010;102:570-576.

1. Gillotin S, iASPP, a potential drug target in cancer therapy. Leukemia Res 2009;33:1175-1177.

22. Laska MJ, Vogel UB, Jensen UB, et al. p53 and PPP1R13L (alias iASPP or RAI) form a feedback loop to regulate genotoxic stress responses. Biochim Biophys Acta 2010;1800:1231-1240.

23. Yin J, Guo L, Wang C, et al. Effects of PPP1R13L and CD3EAP variants on lung cancer susceptibility among nonsmoking Chinese women. Gene 2013;524:228-231.

24. Pinto EM, Musolino NR, Cescato VA, et al. iASPP: A novel protein involved in pituitary tumorigenesis? Front Horm Res 2010;38:70-76.

25. Zhang X, Wang M, Zhou C, et al. The expression of iASPP in acute leukemias. Leukemia Res 2005;29:179-183

26. Wang F, Chang D, Hu FL, et al. DNA repair gene XPD polymorphisms and cancer risk: A meta-analysis based on 56 case-control studies. Cancer Epidemiol Biomarkers Prev 2008;17:507-517.

27. Popp HD, Bohlander SK. Genetic instability in inherited and sporadic leukemias. Genes Chromosome Cancer 2010;49:1071-1081.

28. Allan JM, Smith AG, Wheatley K, et al. Genetic variation in XPD predicts treatment outcome and risk of acute myeloid leukemia following chemotherapy. Blood 2004;104:3872-3877.

29. Hernandez-Boluda JC, Pereira A, Cervantes F, et al. A polymorphism in the XPD gene predisposes to leukemic transformation and new nonmyeloid malignancies in essential thrombocythemia and polycythemia vera. Blood 2012;119:5221-5228.

30. Susini MC, Guglielmelli P, Spolverini A, et al. The ERCC2 Gln/Gln polymorphism at codon 751 is not associated with leukaemic transformation in primary myelofibrosis. Br J Haematol 2013;162:424-427.

31. Poletto V, Villani L, Catarsi P, et al. No association between the XPD Lys751Gln (rs13181) polymorphism and disease phenotype or leukemic transformation in primary myelofibrosis. Haematologica 2013;98: e83-84.

32. Ganster C, Neesen J, Zehetmayer S, et al. DNA repair polymorphisms associated with cytogenetic subgroups in B-cell chronic lymphocytic leukemia. Genes Chromosome Cancer 2009;48:760-767.

33. Chokkalingam AP, Bartley K, Wiemels JL, et al. Haplotypes of DNA repair and cell cycle control genes, $\mathrm{X}$-ray exposure, and risk of childhood acute lymphoblastic leukemia. Cancer Cause Control 2011:22:1721-1730.

34. Batar B, Guven M, Baris S, et al. DNA repair gene XPD and XRCC1 polymorphisms and the risk of childhood acute lymphoblastic leukemia. Leukemia Res 2009;33:759-763.

35. Pakakasama S, Sirirat T, Kanchanachumpol S, et al. Genetic polymorphisms and haplotypes of DNA repair genes in childhood acute lymphoblastic leukemia. Pediatr Blood Cancer 2007;48:16-20.

36. Alvarez-Cubero MJ, Saiz M, Martinez-Gonzalez LJ, et al. Genetic analysis of the principal genes related to prostate cancer: A review. Urol Oncol 2013;31:1419-1429.

37. Sun J, Hsu FC, Turner AR, et al. Meta-analysis of association of rare mutations and common sequence variants in the MSR1 gene and prostate cancer risk. Prostate 2006;66:728-737.

38. Chen Y, Sullivan C, Peng C, et al. A tumor suppressor function of the Msr1 gene in leukemia stem cells of chronic myeloid leukemia. Blood 2011;118:390-400.

39. Xu H, Yang W, Perez-Andreu V, et al. Novel susceptibility variants at 10p12.31-12.2 for childhood acute lymphoblastic leukemia in ethnically diverse populations. J Natl Canc Inst 2013;105:733-742.

40. Seimon TA, Obstfeld A, Moore KJ, et al. Combinatorial pattern recognition receptor signaling alters the balance of life and death in macrophages. Proc Natl Acad Sci U S A 2006;103:19794-19799.

41. Tanaka T, Akira S, Yoshida K, et al. Targeted disruption of the NF-IL6 gene discloses its essential role in bacteria killing and tumor cytotoxicity by macrophages. Cell 1995;80:353-361.

42. Schmidt L, Duh FM, Chen F, et al. Germline and somatic mutations in the tyrosine kinase domain of the MET proto-oncogene in papillary renal carcinomas. Nat Genet 1997;16:68-73.

43. Schutz FA, Pomerantz MM, Gray KP, et al. Single nucleotide polymorphisms and risk of recurrence of renal-cell carcinoma: A cohort study. Lancet Oncol 2013;14:81-87. 